\title{
Magnetic Susceptibility of the Cluster Compounds $\mathrm{Mo}_{6} \mathrm{Se}_{8}$ and $\mathrm{Mo}_{6} \mathrm{Te}_{8}$
}

\author{
G. Concas, F. Congiu, A. Geddo Lehmann, C. Muntoni, S. Sanna, and G. Spano \\ Dipartimento di Fisica, Universitá di Cagliari, and Istituto Nazionale per la Fisica della Materia, \\ S.P. Monserrato-Sestu Km 0.700, I-09042 Monserrato (Cagliari), Italy \\ Reprint requests to Dr. G. C.; Fax: +39 070 510171; E-mail: giorgio.concas@dsf.unica.it
}

Z. Naturforsch. 57 a, 221-225 (2002); received February 18, 2002

\begin{abstract}
The magnetic susceptibility of the cluster compounds $\mathrm{Mo}_{6} \mathrm{Se}_{8}$ and $\mathrm{Mo}_{6} \mathrm{Te}_{8}$ has been measured at temperatures between 82 and $330 \mathrm{~K}$, using a Faraday balance. The paramagnetic and diamagnetic components of the susceptibility have been analyzed. The Pauli component was evaluated using the density of states at the Fermi level obtained by band structure calculations, while the core component was evaluated using the calculated atomic core diamagnetism. The paramagnetic susceptibility of $\mathrm{Mo}_{6} \mathrm{Se}_{8}$ is due mainly to the Pauli contribution, while the Van Vleck contribution is small, in agreement with the metallic feature of the compound. The paramagnetic susceptibility of $\mathrm{Mo}_{6} \mathrm{Te}_{8}$ is due mainly to the Van Vleck term, while the Pauli contribution of the conduction electrons is very small. The result points out that the $\mathrm{Mo}_{6}$ clusters in the telluride retain their molecular character, with small intercluster interactions.
\end{abstract}

Key words: Chalcogenides; Molybdenum; Magnetic Susceptibility; X-ray Diffraction; Chevrel Phases.

\section{Introduction}

The ternary molybdenum chalcogenides known as Chevrel phases are a class of compounds extensively studied in the 1970es for their superconducting properties [1] and more recently as candidates for new thermoelectric materials $[2,3]$. Their general formula is $\mathrm{M}_{x} \mathrm{Mo}_{6} \mathrm{X}_{8}$, in which $\mathrm{X}=\mathrm{S}, \mathrm{Se}$ or Te and $\mathrm{M}$ can be a simple or transition metal atom, or a rare-earth element [4]. The building blocks of the crystal structure are binary $\mathrm{Mo}_{6} \mathrm{X}_{8}$ clusters, in which an octahedron of Mo atoms stays in a cubic $\mathrm{X}_{8}$ cage formed by eight chalcogens. The three dimensional stacking of such clusters has overall rhombohedral symmetry, with $\mathrm{Mo}_{6} \mathrm{X}_{8}$ blocks twisted around the ternary axis, and contains large voids which can be filled with $\mathrm{M}$ metals to give ternary phases. Since the milestone paper of Andersen, Klose, and Nohl of 1978 [5] it was recognized that bonding in filled and unfilled Chevrel phases is mainly due to Mo and $\mathrm{X}$ atoms, with important Mo d - Mo d intracluster interactions and Mo $\mathrm{d}-\mathrm{X} \mathrm{p}$ covalent mixing. Successive studies agree on the basic feature of the overall distribution of the energy levels, i. e. a rough separation of the chalcogen $\mathrm{p}$ and Mo d states below the Fermi level, and the existence of a forbidden gap separating $12 \mathrm{Mo}$ $4 \mathrm{~d}$ states from the remaining 18 Mo $4 \mathrm{~d}$ states $[3,6]$. The Fermi level is just below this gap and lies in very narrow Mo 4d sub-bands which derive from the molecular orbital states of the isolated $\mathrm{Mo}_{6}$ octahedra. In ternary phases, the metallic species M remains weakly coupled to the rest of the structure, with only secondary effects on the electronic properties, modifying the distance between the $\mathrm{Mo}_{6} \mathrm{X}_{8}$ clusters, and therefore Mo $4 \mathrm{~d}$ bandwidth, and providing electrons for band filling. This implies important consequence on the physical properties. For instance, the phases based on rare-earth (RE) $\mathrm{REMo}_{6} \mathrm{~S}_{8}$ and $\mathrm{REMo}_{6} \mathrm{Se}_{8}$ show coexistence of magnetic order and superconductivity [7]. This is possible because the crystal structure is formed by the two nearly-independent sublattices, the first one, made of the $\mathrm{Mo}_{6} \mathrm{X}_{8}$ units, which determines the transport and superconducting properties; the second one, made of the rare-earth atoms, which sustains the long range magnetic order.

The binary Chevrel phases $\mathrm{Mo}_{6} \mathrm{X}_{8}$, in which the metal voids are empty, permit the investigation of the properties of the cluster lattice alone. The aim of this work is to investigate the magnetic susceptibility of the binary Chevrel phases $\mathrm{Mo}_{6} \mathrm{Se}_{8}$ and $\mathrm{Mo}_{6} \mathrm{Te}_{8}$. 
Table 1. X-Ray diffraction data for $\mathrm{Mo}_{6} \mathrm{Se}_{8}$. Relative intensities refer to peak heights. $h k l$ are the rhombohedral Miller indexes and $d_{\exp }$ and $d_{\text {calc }}$ are the experimental and calculated interplanar distances. Errors on the last digit are given in parentheses.

\begin{tabular}{ccrrrc}
$\begin{array}{c}\text { Intensity } \\
\%\end{array}$ & $h$ & $k$ & $l$ & $\begin{array}{c}d_{\text {exp }} \\
\AA\end{array}$ & $\begin{array}{c}d_{\text {calc }} \\
\AA\end{array}$ \\
\hline 100 & 1 & 0 & 0 & $6.64(2)$ & 6.652 \\
21 & 2 & 0 & 0 & $3.330(6)$ & 3.326 \\
16 & 2 & 0 & -1 & $3.016(5)$ & 3.013 \\
24 & 2 & 1 & 0 & $2.938(5)$ & 2.938 \\
23 & 2 & -1 & -1 & $2.765(4)$ & 2.760 \\
45 & 2 & 1 & -1 & $2.732(4)$ & 2.730 \\
14 & 2 & 1 & 1 & $2.650(4)$ & 2.647 \\
9 & 2 & 0 & -2 & $2.394(3)$ & 2.390 \\
5 & 2 & 2 & 0 & $2.316(3)$ & 2.316 \\
43 & 2 & 1 & -2 & $2.250(3)$ & 2.249 \\
12 & 3 & 0 & 0 & $2.218(3)$ & 2.218 \\
19 & 2 & 2 & 1 & $2.157(2)$ & 2.158 \\
22 & 3 & 0 & -1 & $2.126(2)$ & 2.124 \\
11 & 3 & 1 & 0 & $2.084(2)$ & 2.084 \\
80 & 3 & 1 & -1 & $2.011(2)$ & 2.012 \\
9 & 3 & 0 & -2 & $1.874(2)$ & 1.872 \\
15 & 2 & 2 & 2 & $1.862(2)$ & 1.863 \\
21 & 3 & -1 & -2 & $1.800(2)$ & 1.807 \\
\hline
\end{tabular}

\section{Experimental Procedure}

The compounds $\mathrm{Mo}_{6} \mathrm{Se}_{8}$ and $\mathrm{Mo}_{6} \mathrm{Te}_{8}$ can be synthesised by direct combination of the elements. $\mathrm{Mo}_{6} \mathrm{Se}_{8}$ has been prepared using high purity powders of molybdenum reduced in $\mathrm{H}_{2}$ at $800{ }^{\circ} \mathrm{C}$, and grains of selenium. Stoichiometric amounts have been mechanically homogenised and pressed in a hydraulic press. The tablet has been sealed under vacuum in a silica tube and heated at $800^{\circ} \mathrm{C}$ for 2 days, $1000^{\circ} \mathrm{C}$ for 2 days and $1150{ }^{\circ} \mathrm{C}$ for 2 days. The product showed traces of $\mathrm{MoSe}_{2}$ in the X-ray diffraction (XRD) spectrum. Therefore a $2^{\text {nd }}$ thermal treatment has been applied, at $1000{ }^{\circ} \mathrm{C}$ for 1.5 days and at $1200{ }^{\circ} \mathrm{C}$ for 3.5 days, after which the final product appeared homogeneous.

$\mathrm{Mo}_{6} \mathrm{Te}_{8}$ has been prepared using powders of molybdenum, reduced in $\mathrm{H}_{2}$ at $800^{\circ} \mathrm{C}$, and pieces of tellurium. Stoichiometric amounts have been sealed under vacuum in a silica tube; heated at $950{ }^{\circ} \mathrm{C}$ for 11 days and on $1050{ }^{\circ} \mathrm{C}$ for 5 days. The final product was homogeneous at $\mathrm{X}$-ray diffractometric analysis; in particular no peak of metallic molybdenum appeared in the spectrum.

The X-ray diffraction spectra were collected, using a $\theta-2 \theta$ conventional powder diffractometer (Siemens $\mathrm{D} 500)$ and $\mathrm{Cu}-\mathrm{K}_{\alpha}$ radiation.
Table 2. X-Ray diffraction data for $\mathrm{Mo}_{6} \mathrm{Te}_{8}$. Relative intensities refer to peak heights. $h k l$ are the rhombohedral Miller indexes and $d_{\text {exp }}$ and $d_{\text {calc }}$ are the experimental and calculated interplanar distances. Errors on the last digit are given in parentheses.

\begin{tabular}{cccrlc}
\hline $\begin{array}{c}\text { Intensity } \\
\%\end{array}$ & $h$ & $k$ & $l$ & $\begin{array}{c}d_{\text {exp }} \\
\AA\end{array}$ & $\begin{array}{c}d_{\text {calc }} \\
\AA\end{array}$ \\
\hline 71 & 1 & 0 & 0 & $7.01(3)$ & 7.043 \\
37 & 2 & 0 & 0 & $3.524(7)$ & 3.522 \\
12 & 2 & 0 & -1 & $3.212(6)$ & 3.210 \\
62 & 2 & 1 & 0 & $3.092(5)$ & 3.093 \\
79 & 2 & -1 & -1 & $2.943(5)$ & 2.944 \\
36 & 2 & 1 & -1 & $2.901(5)$ & 2.900 \\
11 & 2 & 1 & 1 & $2.769(4)$ & 2.770 \\
10 & 2 & 0 & -2 & $2.548(4)$ & 2.550 \\
6 & 2 & 2 & 0 & $2.434(3)$ & 2.434 \\
42 & 2 & 1 & -2 & $2.400(3)$ & 2.400 \\
8 & 3 & 0 & 0 & $2.348(3)$ & 2.348 \\
34 & 3 & 0 & -1 & $2.258(3)$ & 2.259 \\
14 & 3 & 1 & 0 & $2.195(3)$ & 2.197 \\
100 & 3 & 1 & -1 & $2.133(2)$ & 2.132 \\
4 & 3 & 1 & 1 & $2.064(2)$ & 2.064 \\
14 & 3 & 0 & -2 & $1.997(2)$ & 1.997 \\
14 & 2 & 2 & 2 & $1.946(2)$ & 1.945 \\
44 & 3 & 1 & -2 & $1.915(2)$ & 1.914 \\
\hline
\end{tabular}

The static magnetic susceptibility has been measured with the Faraday method using an RG Cahn electrobalance. The measurements have been performed on powder samples $(150 \mathrm{mg})$ at temperatures ranging from $82 \mathrm{~K}$ to $330 \mathrm{~K}$, with five different maximum magnetic fields, ranging from $0.63 \mathrm{~T}$ to $1 \mathrm{~T}$. The susceptibility was found to be independent on the magnetic field, which rules out the presence of ferromagnetic impurities. The measurements at different temperatures have been performed under helium, using liquid air as cryogenic liquid. The measured susceptibilities were corrected for the diamagnetism of the sample holder.

\section{Results}

Table 1 and Table 2 give the XRD peaks of $\mathrm{Mo}_{6} \mathrm{Se}_{8}$ and $\mathrm{Mo}_{6} \mathrm{Te}_{8}$, indexed in the rhombohedral system. The lattice constants have been refined, using the peak positions, by means of a least squares routine. The rhombohedral parameters for $\mathrm{Mo}_{6} \mathrm{Se}_{8}$ are $a_{\mathrm{r}}=$ $(0.666 \pm 0.001) \mathrm{nm}$ and $\alpha_{\mathrm{r}}=(91.7 \pm 0.1)$ degrees, while $\mathrm{Mo}_{6} \mathrm{Te}_{8}$ has $a_{\mathrm{r}}=(0.706 \pm 0.001) \mathrm{nm}$ and $\alpha_{\mathrm{r}}=$ $(92.5 \pm 0.01)$ degrees. These values agree well with those reported in the literature [8].

Figure 1 shows the magnetic susceptibility of $\mathrm{Mo}_{6} \mathrm{Se}_{8}$ versus temperature, measured between $82 \mathrm{~K}$ 


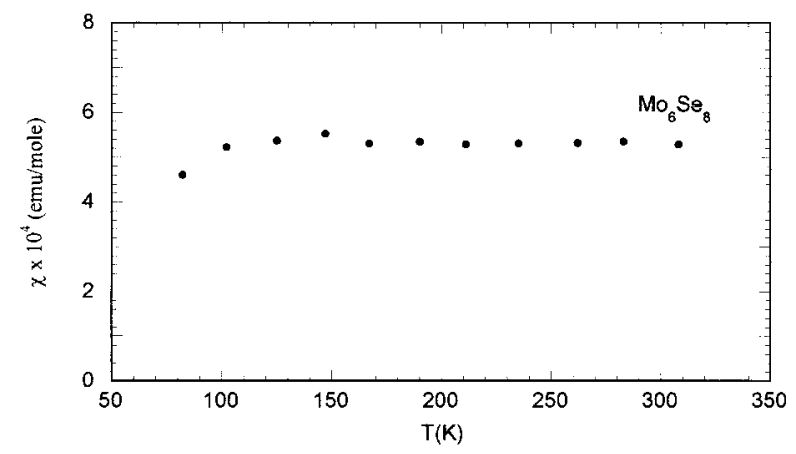

Fig. 1. Magnetic susceptibility of $\mathrm{Mo}_{6} \mathrm{Se}_{8}$ between 82 and $308 \mathrm{~K}$ versus temperature.

and $308 \mathrm{~K}$, corrected for a small Curie-like term due to paramagnetic impurities. The corrected susceptibility shows only a small dependence on temperature near $82 \mathrm{~K}$. The value at room temperature $(298 \mathrm{~K})$ is $(5.28 \pm 0.10) \cdot 10^{-4} \mathrm{emu} / \mathrm{mole}$.

Figure 2 shows the magnetic susceptibility of $\mathrm{Mo}_{6} \mathrm{Te}_{8}$ as a function of temperature, measured between $82 \mathrm{~K}$ and $330 \mathrm{~K}$. It is only weakly temperature-dependent. The value of the susceptibility at room temperature $(297 \mathrm{~K})$ is $(2.59 \pm 0.03)$. $10^{-4} \mathrm{emu} / \mathrm{mole}$.

\section{Discussion and Conclusions}

In the absence of paramagnetic Curie-like terms, the main contributions to the total magnetic susceptibility $\chi_{\text {tot }}$ in a solid are given by

$$
\chi_{\mathrm{tot}}=\chi_{\mathrm{core}}+\chi_{\mathrm{P}}+\chi_{\mathrm{L}}+\chi_{\mathrm{vV}}
$$

where $\chi_{\text {core }}$ is the diamagnetic core component, $\chi_{\mathrm{P}}$ and $\chi_{\mathrm{L}}$ are the Pauli and Landau-Peierls spin susceptibilities of conduction electrons, and $\chi_{\mathrm{VV}}$ indicates the orbital contribution to the paramagnetic susceptibility, analogous to the temuperature-independent Van Vleck paramagnetism of ions in crystal fields.

To separate the various terms appearing in the total susceptibility, we can first of all estimate the expected core diamagnetism, which can be evaluated using the calculated atomic diamagnetism of $\mathrm{Mo}^{+6}, \mathrm{Se}^{-2}$ and $\mathrm{Te}^{-2}$ ions [9]. The core diamagnetic susceptibility has the value of $-4.26 \cdot 10^{-4} \mathrm{emu} / \mathrm{mole}$ for $\mathrm{Mo}_{6} \mathrm{Se}_{8}$ and of $-6.02 \cdot 10^{-4} \mathrm{emu} / \mathrm{mole}$ for $\mathrm{Mo}_{6} \mathrm{Te}_{8}$.

Among the spin terms, in the case of $\mathrm{d}$ electrons, treated in the tight binding approximation, the

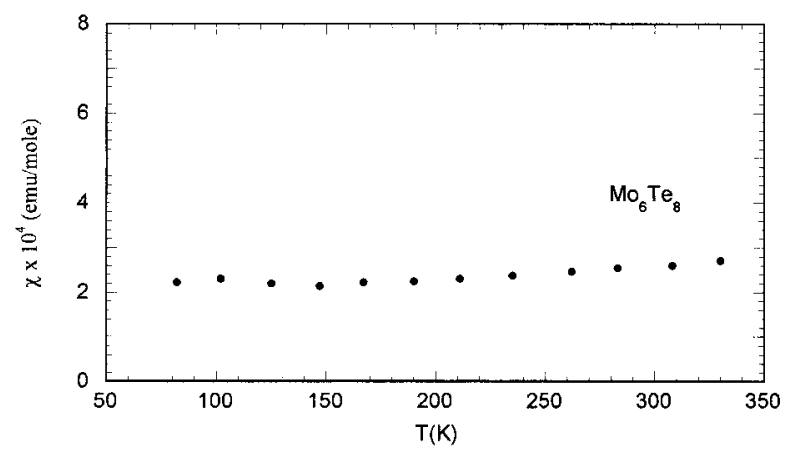

Fig. 2. Magnetic susceptibility of $\mathrm{Mo}_{6} \mathrm{Te}_{8}$ between 82 and $330 \mathrm{~K}$ versus temperature.

Pauli component $\chi_{\mathrm{P}}$ is of prime importance [10]. The Landau-Peierls diamagnetic susceptibility $\chi_{\mathrm{L}}$, which is proportional to $m_{\mathrm{e}} / m^{*}$ and that is equal to $-1 /{ }_{3} \chi_{\mathrm{P}}$ in the case of free electrons [11], can be safely neglected in the present case, owing to the low dispersion of the Mo 4d bands [10].

The Pauli component $\chi_{\mathrm{P}}$ can be estimated from the value of the density of states at the Fermi level $D\left(E_{\mathrm{F}}\right)$ by means of the relation

$$
\chi_{\mathrm{P}}=\mu_{\mathrm{B}}^{2} N D_{\chi}\left(E_{\mathrm{F}}\right)
$$

where $\mu_{\mathrm{B}}$ is the Bohr magneton and $N$ the Avogadro number.

The density of states $D_{\chi}\left(E_{\mathrm{F}}\right)$ appearing in the previous expression is enhanced with respect to the band value $D\left(E_{\mathrm{F}}\right)$ by the elec_tron-elec_tron interaction through the relation

$$
D\left(E_{\mathrm{F}}\right)=D_{\chi}\left(E_{\mathrm{F}}\right) /\left(1+I_{\mathrm{exch}} D_{\chi}\left(E_{\mathrm{F}}\right)\right),
$$

in which $I_{\text {exch }}$ is the electron-electron exchange interaction parameter [12].

The expected Pauli contribution to the measured susceptibility of $\mathrm{Mo}_{6} \mathrm{Se}_{8}$ and $\mathrm{Mo}_{6} \mathrm{Te}_{8}$ can be evaluated from (1) and (2), in which it is reasonable to use for $I_{\text {exch }}$ the value obtained for metallic molybdenum ( $I_{\text {exch }}=0.04 \mathrm{Ry)}$ [5], owing to the fact that the exchange interaction is essentially intra-atomic and that, at $E_{\mathrm{F}}$, the electronic states for $\mathrm{Mo}_{6} \mathrm{X}_{8}$ compounds have essentially Mo character. With the theoretical $D\left(E_{\mathrm{F}}\right)$ of 12.83 states/(spin Mo-atom Ry) [13], we obtain $\chi_{\mathrm{P}}=7.75 \cdot 10^{-4} \mathrm{emu} / \mathrm{mole}$ for $\mathrm{Mo}_{6} \mathrm{Se}_{8}$. If we follow the same procedure for $\mathrm{Mo}_{6} \mathrm{Te}_{8}$, using a value of $D\left(E_{\mathrm{F}}\right)$ of 0.5 states/(spin Mo-atom Ry) [13], we obtain $\chi_{\mathrm{P}}=0.15 \cdot 10^{-4} \mathrm{emu} / \mathrm{mole}$, which is one 
order of magnitude smaller than the measured susceptibility.

By subtracting the estimated $\chi_{\text {core }}$ and $\chi_{\mathrm{P}}$ from the experimental susceptibility at room temperature, we obtain a residual paramagnetic susceptibility of $1.80 \cdot 10^{-4} \mathrm{emu} / \mathrm{mole}$ for $\mathrm{Mo}_{6} \mathrm{Se}_{8}$ and of $8.46 \cdot$ $10^{-4} \mathrm{emu} / \mathrm{mole}$ for $\mathrm{Mo}_{6} \mathrm{Te}_{8}$, indicating that in the selenide the Pauli component is the major term, while in the telluride the orbital paramagnetic contribution $\chi_{\mathrm{vV}}$ is dominant. The previous results reflect the differences in the electronic properties of the two cluster compounds. $\mathrm{Mo}_{6} \mathrm{Se}_{8}$ is a metal and superconductor at $T_{\mathrm{c}}=6.7 \mathrm{~K}$, while $\mathrm{Mo}_{6} \mathrm{Te}_{8}$ is a semimetal and not superconductor. In $\mathrm{Mo}_{6} \mathrm{Se}_{8}$ the Fermi level falls in a peak of the density of states, resulting in a very high $D\left(E_{\mathrm{F}}\right)$ with consequent relatively high critical temperature of superconductivity $T_{\mathrm{c}}$ and high spin paramagnetism above $T_{\mathrm{c}}$, while $E_{\mathrm{F}}$ lies near a valley of the density of states in $\mathrm{Mo}_{6} \mathrm{Te}_{8}[5,6,13]$. This difference can be understood on the basis of an ionic-covalent model for bonding in $\mathrm{Mo}_{6} \mathrm{X}_{8}$ clusters, which assumes that there are covalent interactions between Mo atoms and the chalcogens leading to an increase of the formal electric charge on the $\mathrm{Mo}_{6}$ octahedron [14]. More specifically, only for $\mathrm{X}=\mathrm{S}$ a formal valence of -2 can be assumed for the chalcogen [15], so that the eight sulphur atoms take 16 electrons from the six molybdenum atoms, and 20 electrons per $\mathrm{Mo}_{6}$ unit remain in the Mo d bands (valence electron concentration VEC $=20$ ). In the case of $\mathrm{Se}$ and $\mathrm{Te}$, the formal valences are reduced to 1.8 and -1.7 , respectively [15], leading to $21.60 \mathrm{~d}$ electrons for $\mathrm{Mo}_{6} \mathrm{Se}_{8}$ and of $22.4 \mathrm{~d}$ electrons for $\mathrm{Mo}_{6} \mathrm{Te}_{8}$. The lower $12 \mathrm{Mo} \mathrm{d}$ sub-bands are therefore differently filled in the three $\mathrm{Mo}_{6} \mathrm{X}_{8}$ clusters, a fact which shifts the position of the Fermi level within the bands.

A great deal of information about the band structure would be required to calculate and discuss the paramagnetic term $\chi_{\mathrm{vv}}$, the expression of which in the tight binding approximation may be written as $[10,11]$

[1] $\varnothing$. Fischer and M. B. Maple (eds), Superconductivity in Ternary Compounds, Springer, Berlin 1982.

[2] C. Roche, P. Pecheur, G. Toussaint, A. Jenny, H. Scherrer, and S. Scherrer, J. Phys.: Condensed Matter 10, L333 (1998).

$$
\begin{aligned}
\chi_{\mathrm{VV}}=\int \frac{\mathrm{d} \mathbf{k}}{(2 \pi)^{3}} \sum_{n n^{\prime}} \frac{f\left(E_{n k}\right)-f\left(E_{n^{\prime} \mathbf{k}}\right)}{E_{n^{\prime} k}-\left(E_{n \mathbf{k}}\right.} \\
\cdot \mu_{\mathrm{B}}^{2}\left\langle n \mathbf{k}|L| n^{\prime} \mathbf{k}\right\rangle\left\langle n^{\prime} \mathbf{k}|L| n \mathbf{k}\right\rangle,
\end{aligned}
$$

in which $f\left(E_{n \mathbf{k}}\right)$ and $f\left(E_{n \mathbf{k}}^{\prime}\right)$ are the Fermi functions for electrons in state $n \mathbf{k}$ and $n^{\prime} \mathbf{k}$ with energies $E_{n \mathbf{k}}$ and $E_{n^{\prime} \mathbf{k}}$, respectively, and $L$ is the orbital angular momentum operator. The quantitative evaluation of the orbital paramagnetism in binary $\mathrm{Mo}_{6} \mathrm{Se}_{8}$ and $\mathrm{Mo}_{6} \mathrm{Te}_{8}$ clusters is beyond the aim of this work. However, among the few attempts which have been made at correlate expression (3) to a limited number of crucial band and bonding parameters, we would like to mention the one connecting $\chi_{\mathrm{VV}}$ and bond character in complex diamond-like semiconductors [16, 17]. In that case the orbital paramagnetism is expressed as $\chi_{\mathrm{VV}}^{0}(1-\lambda)$, in which the covalency parameter $(1-\lambda)$ varies in the range 1 to 0 from ionic to covalent compounds, and $\chi_{\mathrm{VV}}^{0}$ is the paramagnetic susceptibility in the case of purely exchange interactions. Following the idea of Pauling [18], the degree of ionicity of a bond, denoted by $\lambda$, can be related to the electronegativity difference $\Delta x$ between the interacting atoms. For Mo-Se and Mo-Te bonds one has $\Delta x^{\mathrm{Se}}=(2.4$ $-1.8)=0.6$ and $\Delta x^{\mathrm{Te}}=(2.1-1.8)=0.3$, so that an increase of $\chi_{\mathrm{VV}}$ can be expected from $\mathrm{Mo}_{6} \mathrm{Se}_{8}$ to $\mathrm{Mo}_{6} \mathrm{Te}_{8}$, because the ionicity of the Mo-X bond decreases as one goes from the selenide to the telluride. It can be speculated that in the case of compounds containing $\mathrm{Mo}_{6}$ clusters the quantity $\chi_{\mathrm{VV}}^{0}$ can be identified with the orbital paramagnetic susceptibility of the isolated $\mathrm{Mo}_{6}$ octahedron, in which 24 valence electrons saturate 12 two-centred metal-metal bonds. The high value of $\chi_{\mathrm{VV}}$ found in $\mathrm{Mo}_{6} \mathrm{Te}_{8}$ therefore reflects the fact that in the telluride the $\mathrm{Mo}_{6}$ octahedra retain strongly their molecular character, with minimum transfer of electrons from Mo to the chalcogen atoms.

\section{Acknowledgements}

We would like to dedicate this work to the memory of Professor Pietrino Manca.

[3] R. W. Nunes, I. I. Mazin, and D. J. Singh, Phys. Rev. B59, 7969 (1999).

[4] R. Chevrel and M. Sergent, J. Solid State Chem. 6, 433 (1973). 
[5] O. K. Andersen, W. Klose, and H. Nohl, Phys. Rev. B17, 1209 (1978).

[6] Shu-Hui Cai and Chu-Wan Liu, J. Chem. Soc. Faraday Trans. 91, 479 (1995).

[7] O. Pena, F. LeBerre, M. Sergent, R. Horyn, and A. Wojakowski, Physica C 235-240, 771 (1994).

[8] R. Chevrel and M. Sergent, in [1].

[9] R. R. Gupta, in K. H. Hellwege and A. M. Hellwege (eds), Landolt-Boernstein Vol. II/16, Springer, Berlin 1986.

[10] A. M. Klogston, W. Jaccarino, and Y. Yafet, Phys. Rev. 134, A650 (1964).

[11] H. J. Zeiger and G. W. Pratt, Magnetic Interactions in Solids, Clarendon Press, Oxford 1973.
[12] P. Fazekas, Lecture Notes on Electron Correlation and Magnetism, World Scientific, Singapore 1999.

[13] H. Nohl, W. Klose, and O. K. Andersen, in [1].

[14] K. Yvon, A. Paoli, R. Flükiger, and R. Chevrel, Acta Crystallogr. B33, 3066 (1977).

[15] K. Yvon in [1].

[16] L. Garbato, P. Manca, and A. Spiga, Nature Physical Science 239, 156 (1972).

[17] L. Garbato and P. Manca, Chem. Phys. Letters 48, 151 (1977).

[18] L. Pauling, The Nature of the Chemical Bond, $3^{\text {rd }}$ ed., Cornell University Press, Ithaka 1960. 